\title{
1991. Effect of sink flow on dual-valve electro-hydraulic excitation system
}

\author{
Zhen Meng ${ }^{1}$, Chuan-yu $\mathrm{Wu}^{2}$, Jing $\mathrm{Ni}^{3}$ \\ ${ }^{1,2}$ Zhejiang Sci-Tech University, School of Mechanical Engineering and Automation, Hangzhou, China \\ ${ }^{3}$ Hangzhou Dianzi University, School of Mechanical Engineering, Hangzhou, China \\ ${ }^{2,3}$ Corresponding author \\ E-mail: ${ }^{1}$ mengzhen0601@126.com, ${ }^{2}$ cywu@zstu.edu.cn, ${ }^{3}$ nj2000@hdu.edu.cn \\ Received 28 October 2015; received in revised form 10 January 2016; accepted 18 January 2016 \\ DOI http://dx.doi.org/10.21595/jve.2016.16589
}

\begin{abstract}
In this study, the main goal is to investigate the displacement gain characteristics of novel dual-valve Electro-Hydraulic Excitation system (EHES). Relative to conventional system, the dual-valve system can improve the amplitude range, but the range is always affected by some nonlinear factors. In order to analysis on the issue, a nonlinear mathematical model of dual-valve EHES is established based on Bernoulli and classical electro-hydraulic servo equation. Then, the results of numerical simulations by using of the MATLAB/Simulink software are compared with experimental results. Simulation and experimental results show that the sink flow mainly influences on the flow dynamic characteristics of dual-valve EHES. Because of that, dual-valve EHES only can improve $50 \%-70 \%$ of displacement within 5 to $50 \mathrm{~Hz}$ of excitation frequency and $45 \%-80 \%$ of displacement amplitude within $20 \%$ to $100 \%$ of command value.
\end{abstract}

Keywords: dual-valve EHES, vibration exciter, effect of sink flow, amplitude gain.

\section{Introduction}

The Electro-Hydraulic Excitation system (EHES) is a key component for modern simulation test of automobile or mobile machines and the seismic fatigue test of dams or tall buildings. The system is mainly worked by the reciprocating motion of valve spool, which varies the flow in cycles (Fig. 1). However, the vibration amplitude is restricted within a narrow range due to the characteristic of valve port [1]. Therefore, the optimization of vibration performance for EHES has been the focus of both academic and industrial field.

In conventional EHES, it usually adopts slide or nozzle flapper valve to control the hydraulic cylinder or torque motor [2]. In order to improve the response characteristics of the hydraulic valve, Han et al., Zhang et al., Lian et al. and Wang et al. replaced the slide valve with rotary valve [3-6]. Liu et al. and Ruan et al. proposed a special valve structure with two degree of freedom $[7,8]$. For purpose of optimizing the output waveform, a series of controller was designed by introducing Lyapunov function method, self-adjustment quantitative feedback theory, varying error controls strategy, neurodynamic optimization and other mathematical method [9-16]. For the sake of researching the vibration performance, Han et al. simulated the vibration characteristics of a new electro-hydraulic vibrator by using the AMESim software [17]. Wei et al. and Kou et al. summarized the law of pressure pulsation [18, 19]. However, the studies all above have only focused on the frequency range and stability of the excited system, and ignored the displacement amplitude range. A little paper introduced the unique advantages of multi-valve system in enhancing displacement amplitude ranges. Ren et al. proposed the offset valve [20]. Chiang et al. applied the parallel switching valve to control the vertical vibration system [21]. But they only focused on the application effect, lacking studies on the excitation mechanism. In view of this, this article will introduce a novel EHES with dual-valve in order to enhance the vibrator performance, and investigate the vibration characteristics of the system, especially in the aspect of displacement amplitude.

In the first part of the paper, a mathematical model of dual-valve EHES is presented. The system is mainly described by Bernoulli and classical flow characteristic equation. Then, the effect of sink flow on displacement gain characteristic of the system within various vibration parameters 
is analyzed by using the MATLAB/Simulink software. Meanwhile, the test platform is set up to validate the calculation results. The simulation and experimental data are presented and discussed in third part. Finally, the conclusions are given from a number of comparisons between the experimental and the numerical results.

\section{Dual-valve EHES model}

The dual-valve EHES is shown in Fig. 2, which consists of two parallel servo valves and a vibration cylinder. Similar to conventional system, the dual-valve system also relay on the cyclic flow to achieve the continuous reciprocating vibrations. For the purpose of improving the displacement amplitude, the valves must be controlled by the same exciting signal.

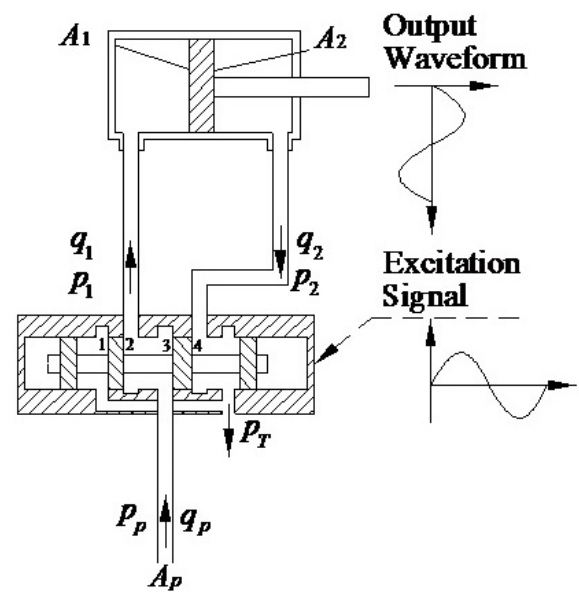

Fig. 1. Electro-hydraulic excitation system

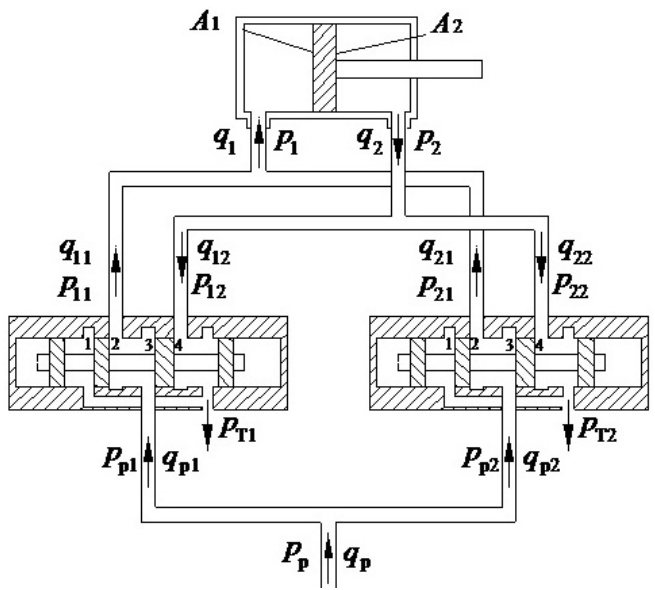

Fig. 2. Dual-Valve EHES

\subsection{Valve port flow}

As shown in Fig. 2, the valve spool will move left when the excitation signal is positive, and the high pressure oil will flow into the left cavity of cylinder. In contrast, the high pressure oil will flow into right cavity when the signal is negative. Therefore, the flow equation of valves is:

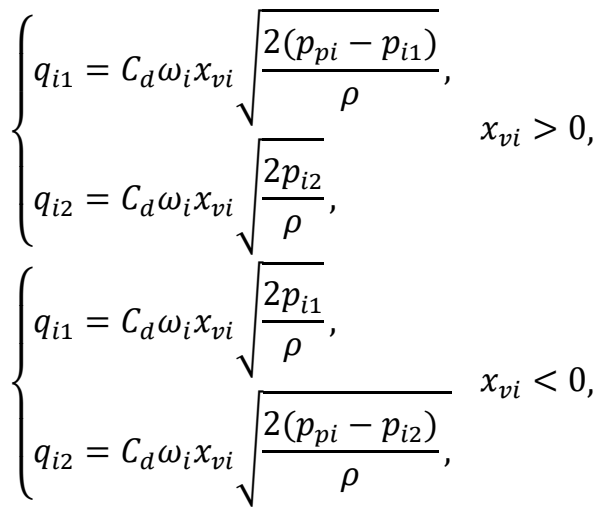

where $p_{p i}$ is the supply pressure of $i$ th valve, $p_{i 1}$ and $p_{i 2}$ is the pressure of flowing out and in $i$ th valve. $q_{i 1}$ and $q_{i 2}$ is the flow out and in $i$ th valve $(i=1,2)$. 


\subsection{Sink flow model}

As shown in Fig. 2, sink flow will appear on the tee joint when the oil flows to cylinder. According to Bernoulli's equation:

$$
\begin{aligned}
& \left\{\begin{array}{l}
p_{11}+0.5 \rho\left(\frac{q_{11}}{A_{11}}\right)^{2}=p_{1}+0.5 \rho\left(\frac{q_{1}}{A_{1}}\right)^{2}, \\
p_{21}+0.5 \rho\left(\frac{q_{21}}{A_{21}}\right)^{2}=p_{1}+0.5 \rho\left(\frac{q_{1}}{A_{1}}\right)^{2},
\end{array}\right. \\
& \left\{\begin{array}{l}
p_{12}+0.5 \rho\left(\frac{q_{12}}{A_{12}}\right)^{2}=p_{2}+0.5 \rho\left(\frac{q_{2}}{A_{2}}\right)^{2}, \\
p_{22}+0.5 \rho\left(\frac{q_{22}}{A_{22}}\right)^{2}=p_{2}+0.5 \rho\left(\frac{q_{2}}{A_{2}}\right)^{2},
\end{array}\right.
\end{aligned}
$$

where $p_{1}$ and $p_{2}$ is the pressure of the left and right cavity. The flow into and out of the cylinder is $q_{1}$ and $q_{2} . A_{1}$ is the area of piston, and $A_{2}$ is the cross sectional area of right cavity. $A_{i 1}$ and $A_{i 2}$ are the cross sectional area of pipeline $(i=1,2)$ :

$$
\begin{aligned}
& \begin{cases}q_{1}=\left(C_{d} \omega_{1} x_{v 1} \sqrt{\frac{2\left(p_{p}-p_{1}\right)}{\rho}}+C_{d} \omega_{2} x_{v 2} \sqrt{\frac{2\left(p_{p}-p_{1}\right)}{\rho}}\right), & x_{v 1}>0, \\
q_{2}=\left(C_{d} \omega_{1} x_{v 1} \sqrt{\left.\frac{2 p_{2}}{\rho}+C_{d} \omega_{2} x_{v 2} \sqrt{\frac{2 p_{2}}{\rho}}\right),}\right. & x_{v 2}>0,\end{cases} \\
& \begin{cases}q_{1}=\left(C_{d} \omega_{1} x_{v 1} \sqrt{\left.\frac{2 p_{1}}{\rho}+C_{d} \omega_{2} x_{v 2} \sqrt{\frac{2 p_{1}}{\rho}}\right),}\right. & x_{v 1}<0, \\
q_{2}=\left(C_{d} \omega_{1} x_{v 1} \sqrt{\frac{2\left(p_{p}-p_{2}\right)}{\rho}}+C_{d} \omega_{2} x_{v 2} \sqrt{\frac{2\left(p_{p}-p_{2}\right)}{\rho}}\right), & x_{v 2}<0 .\end{cases}
\end{aligned}
$$

The first equation of Eq. (3) multiplies by $q_{11}$, and the second one multiplies by $q_{21}$. Then, substituting Eq. (1) into Eq. (3) yields Eq. (5). The first equation of Eq. (4) multiplies by $q_{12}$, and the second one multiplies by $q_{22}$. Then, substituting Eq. (1) into Eq. (4) yields Eq. (6). Due to the connection pipe is hard and short, the energy loss can be neglected. Therefore, the sink flow equation can be expressed as follow.

\subsection{Dual-valve coupling model}

According to Fig. 2, the flow into and out of cylinder can be expressed by:

$$
\left\{\begin{array}{l}
q_{1}=C_{n p}\left(p_{1}-p_{2}\right)+C_{e p} p_{1}+\frac{V_{1}}{\beta_{e}} \dot{p}_{1}+\dot{V}_{1}, \\
q_{2}=C_{n p}\left(p_{1}-p_{2}\right)-C_{e p} p_{2}-\frac{V_{2}}{\beta_{e}} \dot{p}_{2}+\dot{V}_{2} .
\end{array}\right.
$$

According Newton's second law, the equilibrium equation of piston is given by:

$$
A_{1} p_{1}-A_{2} p_{2}=m \ddot{x}_{p}+B_{p} \dot{x}_{p}+F_{L}
$$


Combing Eqs. (5)-(8), the dual-valve EHES are defined mathematically as follows:

$$
\left\{\begin{array}{l}
K C_{d} \omega\left(x_{v 1}+x_{v 2}\right) \sqrt{\left(\alpha_{1} p_{p}+\alpha_{2} p_{L}\right)}=\frac{L_{s} A_{1}\left(1+\eta^{4}\right)}{2\left(1+\eta^{3}\right) \beta_{e}} \dot{p}_{L}+A_{1} \dot{x}_{p} \\
A_{1} p_{L}=m \ddot{x}_{p}+B_{p} \dot{x}_{p}
\end{array}\right.
$$

where:

$K=\sqrt{\frac{2}{\rho\left(1+\eta^{3}\right)}}$

$\alpha_{1}= \begin{cases}1, & x_{v 1}>0, x_{v 2}>0, \\ \frac{\eta^{3}}{\left(1+\eta^{2}\right)^{2}}, & x_{v 1}<0, \quad x_{v 2}<0,\end{cases}$

$\alpha_{2}= \begin{cases}-1, & x_{v 1}>0, x_{v 2}>0, \\ \frac{1}{\left(1+\eta^{2}\right)^{2}}, & x_{v 1}<0, \quad x_{v 2}<0 .\end{cases}$

\section{Simulation and experiment}

\subsection{Simulation test}

The simulations have two parts. Part I: it is mainly to investigate the effect of sink flow on characteristics of output displacement. Firstly, the characteristics of the system with sink flow are numerically calculated by using Matlab/Simulink software. Then, the results are compared with the output displacement of the model without sink flow. Part II: it is mainly to analysis the relationship between the sink flow and the exciting parameters. Firstly, it is to simulate and analysis the output displacement of dual-valve EHES with and without sink flow under various excitation frequencies. Then, the results are compared with the output displacement under various command values of valve (valve opening).

Especially, the model with and without sink flow are simulated under the same condition. The main parameters in the simulation are shown in Table 1.

Table 1. Main parameter of the system

\begin{tabular}{|l|c|c|c|}
\hline \multicolumn{1}{|c|}{ Parameter } & Symbol & Value & Unit \\
\hline Voltage Gain & $K_{v}$ & 0.1 & $\mathrm{~mm} / \mathrm{V}$ \\
\hline Valve port area of gradient & $W$ & 5.65 & $\mathrm{~mm}$ \\
\hline Valve flow coefficient & $C_{d}$ & 0.6 & \\
\hline Working pressure & $p_{p}$ & 6 & $\mathrm{MPa}$ \\
\hline Oil density & $\rho$ & 900 & $\mathrm{~kg} / \mathrm{m}^{3}$ \\
\hline Piston diameter & $A_{1}$ & 60 & $\mathrm{~mm}^{2}$ \\
\hline Rod diameter & $A_{2}$ & 35 & $\mathrm{~mm}^{2}$ \\
\hline Cylinder stroke & $L_{s}$ & 100 & $\mathrm{~mm}$ \\
\hline Asymmetric ratio & $H$ & 0.583 & \\
\hline Oil elastic modulus & $\beta_{e}$ & 700 & $\mathrm{MPa}$ \\
\hline Viscous damping coefficient & $B_{p}$ & 300 & $\mathrm{~N} \cdot \mathrm{s} / \mathrm{m}$ \\
\hline Total mass & $M$ & 10 & $\mathrm{Kg}$ \\
\hline Vibration frequency & $f$ & $5-50$ & $\mathrm{~Hz}$ \\
\hline Command value of valve & $x_{v}$ & $20,40,60,80,100$ & $\%$ \\
\hline
\end{tabular}

According to the equations above, the dual-valve EHES model without sink flow can be simply considered the superposition of single valve system. And, the single valve EHES can be expressed 
by:

$$
\left\{\begin{array}{l}
C_{d} \omega x_{v} \sqrt{\frac{2\left(p_{p}+\lambda p_{L}\right)}{\rho\left(1+\eta^{3}\right)}}=\frac{L_{s} A_{1}\left(1+\eta^{4}\right)}{2\left(1+\eta^{3}\right) \beta_{e}} \dot{p}_{L}+A_{1} \dot{x}_{p} \\
A_{1} p_{L}=m \ddot{x}_{p}+B_{p} \dot{x}_{p}
\end{array}\right.
$$

where, if $x_{v}>0$, then $\lambda=-1 ; x_{v}<0$, then $\lambda=1$.

In order to intuitively reflect the effect of sink flow on the system, this paper introduce the bias gain, which is defined as follow:

$\operatorname{gain}_{e}=\operatorname{gain}_{s}-\operatorname{gain}_{d}$

where the gian $_{s}$ is the displacement gain without sink flow. The gian ${ }_{d}$ is the gain with sink flow. And the gian $_{s}$ can be expressed as follow:

$\operatorname{gain}_{s}=\frac{x_{p s}-x_{s}}{x_{s m}}$

where $x_{p s}$ is the displacement of the system without sink flow. $x_{s}$ is the displacement of single valve model. $x_{s m}$ is the maxim value of $x_{s}$.

The gain $_{d}$ can be expressed by:

$\operatorname{gain}_{d}=\frac{x_{p d}-x_{s}}{x_{s m}}$

where $x_{p d}$ is the displacement of the system with sink flow.

\subsection{Experimental test}

In order to verify the simulation results, the experimental tests also have two parts, and the goal of each part is the same as the simulation. As shown in the Figs. 3-4, the experimental platform of dual-valve EHES has been developed and designed, which is divided into three modules: excitation controller, vibration generator and measuring instrument. The controller sent the same signals to dual valve at the same time, which used is S7-300 PLC produced by SIMENS, and it is programmed by PCC using STEP 7 software. The generator, including servo valves and excitation cylinder, excites vibration waveform following the signals. The servo valves used are 4WSE2EM6 produced by Rexroth, which are controlled by the voltage signal. The excitation cylinder is special customized by referencing the CD $210 \mathrm{~B} 63 / 35-100$. The instrumentation is mainly measuring for vibration displacement. The measuring instrument is VIBXPERT II VIB5.325 produced by PRUFTECHNIK, and the vibration sensor is VIB 6.142R, which is mounted on the top of the piston rod by magnetic base. And the specifications of the system are summarized in Table 2.

Table 2. Experimental instruments

\begin{tabular}{|l|c|c|c|}
\hline \multicolumn{1}{|c|}{ Instrument } & Model & Range & Signal \\
\hline Servo valve & 4WSE2EM6 & $25 \mathrm{~L} / \mathrm{min}$ & $\pm 10 \mathrm{~V}$ \\
\hline Program controller & $\begin{array}{c}\text { S7-300 } \\
\text { CPU 314 }\end{array}$ & - & - \\
\hline A/D module & SM332 & \pm 27648 & $\pm 10 \mathrm{~V}$ \\
\hline FFT data collector and signal analyzer & VIB5.325 & $10 \mathrm{KHz}$ & - \\
\hline Mobile accelerometer & VIB 6.142R & & \\
\hline
\end{tabular}


To compare the simulation results, the experimental parameters are the same and are shown in Table 1.

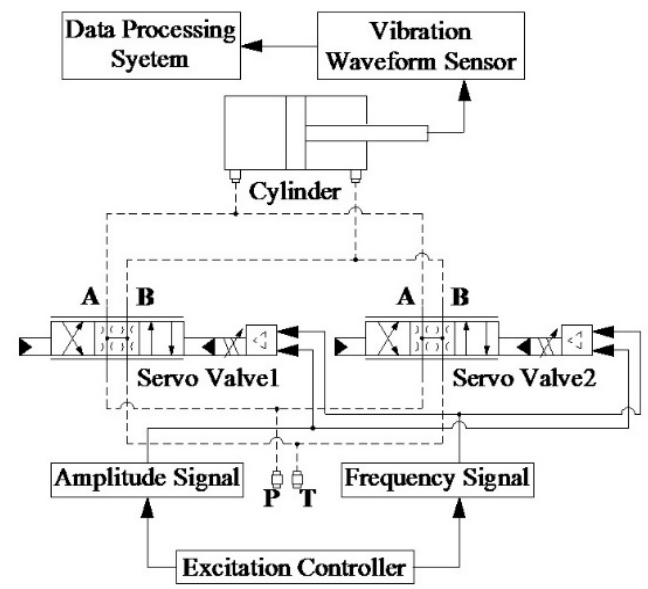

Fig. 3. EHES controlled by dual-valve

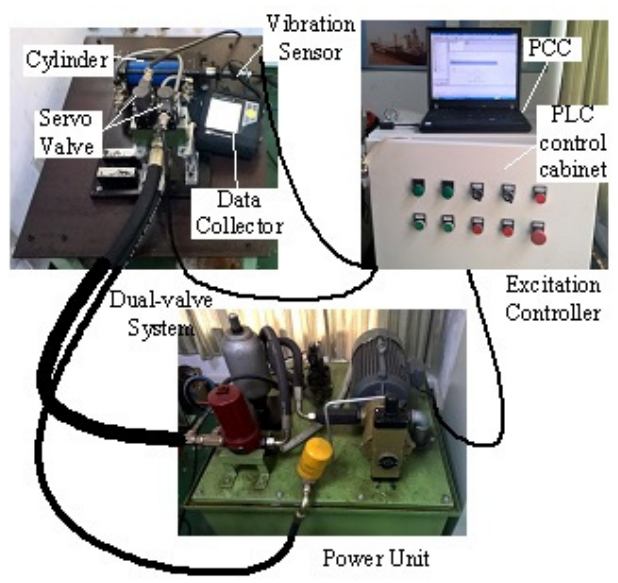

Fig. 4. Experiment system

\section{Results and discussion}

Fig. 5 shows the amplitude gain of displacement from simulation part I. It can be seen that the gains without sink flow by numerically simulation are almost from -1 to 1 . And the gains with sink flow are only from -0.52 to 0.52 . It indicates that the sink flow restricts the ability of dual-valve EHES on enhancing displacement range. As can be seen from Fig. 5, the bias gains are obviously asymmetry in one period. The characteristic of asymmetry means the sink flow lead to saturated pressure appearing earlier than that of the system without sink flow when piston moving to the end of cylinder. Therefore, the sink flow mainly affects the characteristics of cavity pressure by simulation analysis.

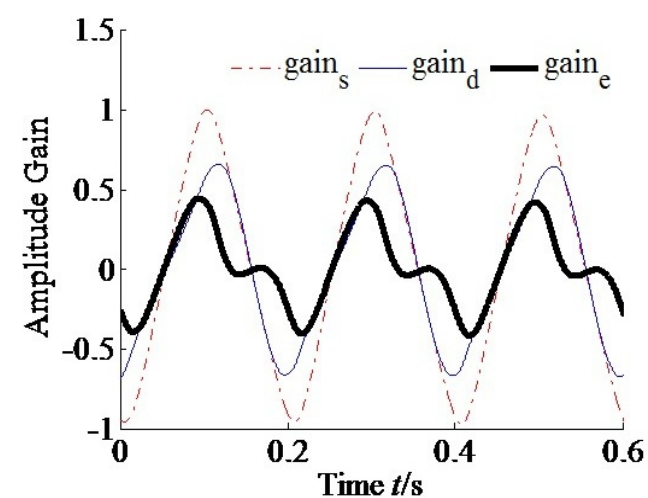

Fig. 5. Simulation results

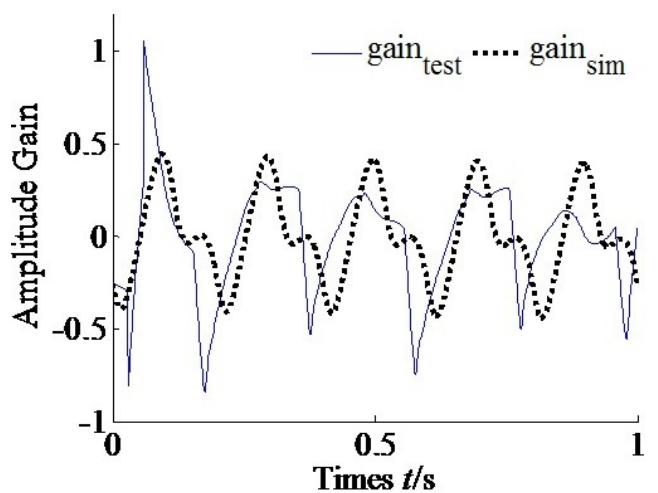

Fig. 6. Experimental results

As Fig. 6 shown, the curve gain $_{\text {sim }}$ represents the simulation results of bias gain. The curve gain $_{\text {test }}$ represents the experimental results. By comparing two curves, it is shown that the experimental data have been validated the simulation results. The asymmetry characteristics of

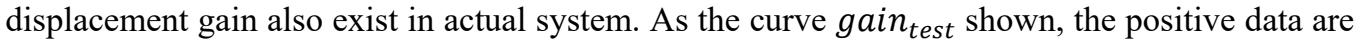
more smoothly, and the negative data are more acute. It means that the sink flow characteristic more obvious on small cavity of cylinder than big. That is the smaller volume of cavity, the sink flow effect more obvious.

Therefore, based on the results of test part I, the conclusion is that the sink flow affects mainly 
on the pressure characteristics of cylinder, and then restricts the flow of dual-valve EHES. Thus, the dual-valve EHES cannot further enhance displacement range.

Fig. 7(a) and (b) show the bias gain of displacement under various frequencies from test part II. Fig. 8 shows the peak values of bias gain. As Fig. 7(a) and (b) shown, it can be seen that the trend of experimental data is similar to the simulation results in a certain period. The bias gain would not vanish as excitation frequency change. As Fig. 8 shown, the curve of simulation results fluctuates smoothly, but the curve of experimental data rise slowly. The peak values of bias gain are steady in general. It means the excitation frequencies have effect on bias gain, but the effect is not very significant. Thus, the effect of sink flow on displacement gain under various frequencies is not obvious. The reason is that the high frequency movement of spool induces the pressure transient on valve port. In fact, the characteristic of pressure would exhibit the displacement amplitude range of single-valve system. But it has a relatively minimal impact on sink flow.

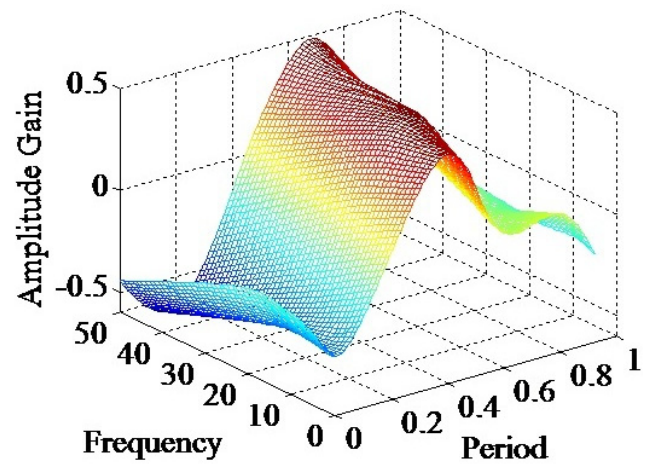

a)

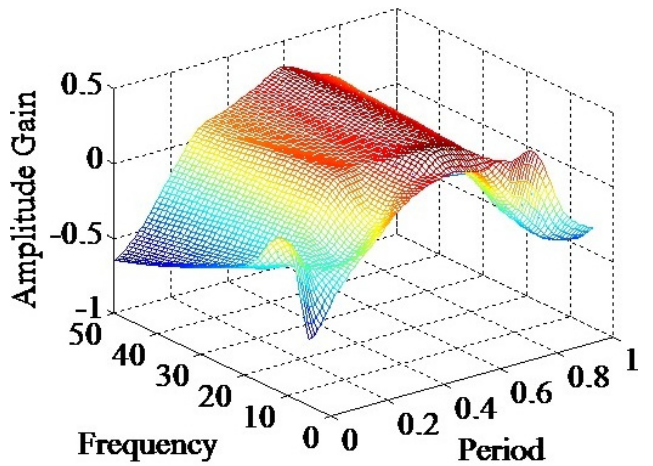

b)

Fig. 7. Bias gain with varying frequency

As Fig. 8 shown, the peak values of experimental data are generally smaller than simulation results, which just vary from 0.28 to 0.34 . It means the mathematic model of dual-valve EHES only can explain the trend of the dynamic characteristic of bias gain under various frequencies, but not quantitative analysis of actual displacement gain. The reason is that the actual system has more nonlinear and uncertainty parameters than simulation model.

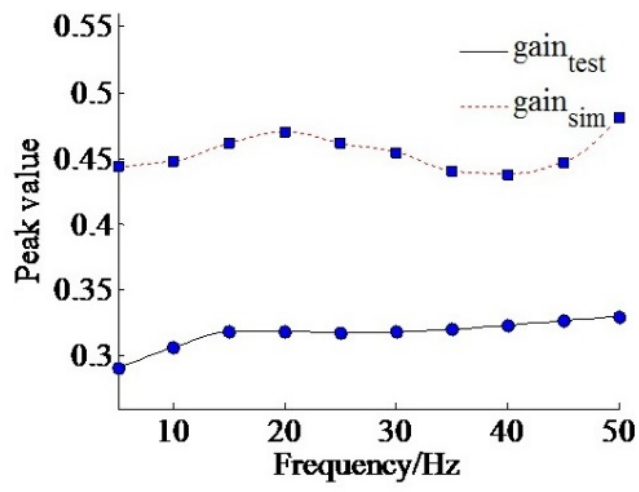

Fig. 8. Peak gain with varying frequency

Figs. 9(a) and (b) show the bias gain under various command value of valve from test part II. Fig. 10 shows the peak values of bias gain arise with varying command value. As Fig. 9(a) and (b) shown, it can be seen that the trend of experimental data is also similar to the simulation results. The bias gain would not vanish as command value change as well. Therefore, the sink flow is the generic characteristics of dual-valve EHES. As Fig. 10 shown, the curve of simulation results rises 
from 0.3 to 0.46 , and the curve of experimental data rises from 0.2 to 0.37 . It means that the more command value, the bias gain more obvious. The reason is that the more command value of valve induces the more flow on valve port. And the characteristics of flow have a relatively obvious impact on sink flow comparing to the results under various frequencies. Otherwise, as Fig. 10 shown, the experimental data are also smaller than simulation results.

Therefore, the command values of valve mightily influence the bias gain comparing to excitation frequency. That is the characteristic of flow directly affects the sink flow; and then it further restricts the displacement gain of the dual-valve system. But the command values can promote the displacement amplitude of single-valve system increase. In a word, sink flow influence on the displacement gain of dual-valve system. But the varying excitation parameters can affect both the bias gain and the output amplitude of the system. Thus, the excitation parameters must be chosen reasonably to reduce sink flow influence and achieve a better performance on the output displacement of the dual-valve system.

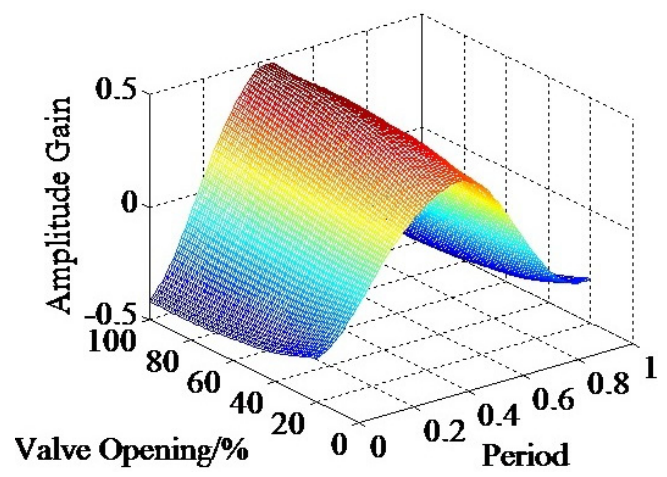

a)

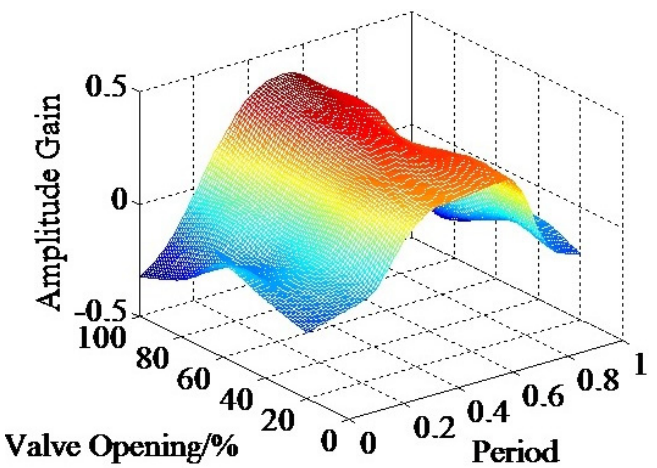

b)

Fig. 9. Bias gain with varying command value

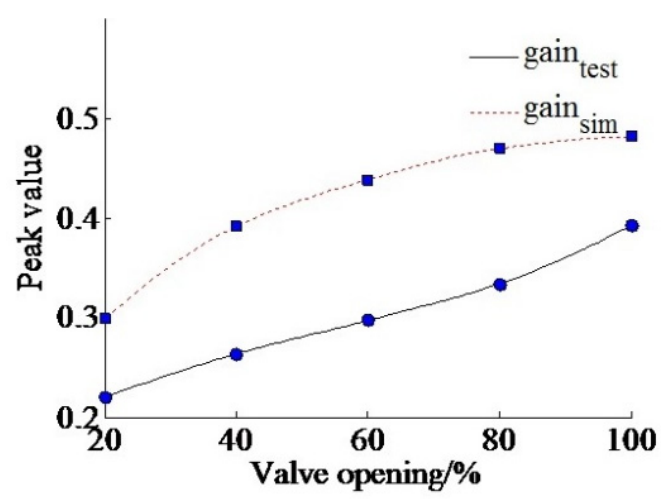

Fig. 10. Peak gain with varying command value

\section{Conclusion}

From the experimental data and simulation results, the conclusions are summarized as follows:

1) A mathematical model for studying the sink flow effect of dual-valve EHES has been presented. Considering the simulation and test results, the dual-valve EHES only can improve $50 \%-80 \%$ displacement ranges compared to the system without sink flow under pressure $6 \mathrm{MPa}$.

2) The sink flow affects mainly on the pressure characteristics of cylinder, and then restricts the ability of dual-valve EHES on enhancing displacement range.

3) The bias gain, which intuitively reflects the effect of sink flow on the system, varies from 0.29 to 0.34 when the excitation frequencies are from $5 \mathrm{~Hz}$ to $50 \mathrm{~Hz}$, and it varies from 0.2 to 0.38 
when the command value are from $20 \%$ to $100 \%$.

4) The experimental results suggest that the command value have more influence on bias gain. That is the command value directly affects the sink flow, and restricts the displacement range of dual-valve EHES.

5) The excitation parameters should be properly matched to reduce sink flow influence.

\section{Acknowledgements}

This research was supported by the National Natural Science Foundation of China (Grant No. 51375129), the 521 Talent Training Program of Zhejiang Sci-Tech University.

\section{References}

[1] Saton N. Frequency characteristics of electro-hydraulic vibrator. Journal of UOEH, Vol. 24, Issue 4, 2002, p. 405-412.

[2] Ghafari S. H., Golnaraghi F., Ismail F. Effect of localized faults on chaotic vibration of rolling element bearings. Nonlinear Dynamics, Vol. 53, 2008, p. 287-301.

[3] Han D., Gong G. F., Yang H. Y., et al. Waveforms analysis and optimization of new electro-hydraulic excitation technology. Journal of Central South University, Vol. 21, 2014, p. 3098-3106.

[4] Zhang J. Z., Guan L. Z., Li K. K. Study on high frequency hydraulic exciter based on rotary valve control. Advanced Materials Research, Vol. 490, Issue 495, 2012, p. 1441-1445.

[5] Lian H. Z., Kou Z. M. Characteristics analysis and development summary on hydraulic exciting ways of vibrating machine. Coal Mine Machinery, Vol. 27, Issue 11, 2007, p. 12-14.

[6] Wang S. H., Tsung T. T., Han L. L. Hydraulic square-wave pressure generator with a specific rotating valve. Measurement, Vol. 42, 2009, p. 672-677.

[7] Liu Y., Gong G. F., Yang H. Y., et al. Mechanism of electro-hydraulic exciter for new tamping device. Journal of Central South University, Vol. 21, 2014, p. 511-520.

[8] Ruan J., Burton R. T. An electrohydraulic vibration exciter using a two-dimensional valve. Proceedings of the Institution of Mechanical Engineers, Part I: Journal of Systems and Control Engineering, Vol. 223, 2009, p. 135-147.

[9] Yu J., Zhuang J., Yu D. H. State feedback integral control for a rotary direct drive servo valve using a Lyapunov function approach. ISA Transactions, Vol. 54, 2015, p. 207-217.

[10] Kyoung K. A., Quang T. D. Self-tuning of quantitative feedback theory for force control of an electro-hydraulic test machine. Control Engineering Practice, Vol. 17, 2009, p. 1291-1306.

[11] Guan G. F., Cong D. C., Han J. W., et al. Application of FRF estimator based on errors-in-variables model in multi-input multi-output vibration control system. Chinese Journal of Mechanical Engineering, Vol. 4, Issue 20, 2007, p. 101-105.

[12] Weng F. L., Mao W. J. Parameter-dependent vibration-attenuation controller design for electro-hydraulic actuated linear structural systems. Earthquake Engineering and Engineering Vibration, Vol. 11, 2012, p. 73-82.

[13] Peng Y. G., Wang J., Wei W. Model predictive control of servo motor driven constant pump hydraulic system in injection molding process based on neurodynamic optimization. Journal of Zhejiang University - Science C (Computers and Electronics), Vol. 15, Issue 2, 2014, p. 139-146.

[14] Jia W. A., Ruan J., Ren Y. Separate control of high frequency electro-hydraulic vibration exciter. Chinese Journal of Mechanical Engineering, Vol. 2, Issue 24, 2011, p. 293-302.

[15] Jarosław K., Marek S., Waldemar R. The control system for a vibration exciter. Solid State Phenomena, Vol. 198, 2013, p. 600-605.

[16] Marko Š., Mihael D., Niko H. Modelling of hydraulic spool-valves with specially designed metering edges. Strojniški Vestnik - Journal of Mechanical Engineering, Vol. 60, Issue 2, 2014, p. 77-83.

[17] Han D., Gong G. F., Liu Y. AMESim based numerical analysis for electrohydraulic exciter applied on new tamper. Applied Mechanics and Materials, Vols. 190-191, 2012, p. 11-18.

[18] Wei X. Y., Kou Z. M., Lu Z. R. Analysis on the pressure fluctuation law of a hydraulic exciting system with a wave-exciter. International Journal of Plant Engineering and Management, Vol. 4, Issue 16, 2011, p. 208-214. 
[19] Kou Z. M., Zhang H. X., Wu J., et al. Numerical simulation and experimental analysis of an active hydraulic excitation system based on pulsating flow. Advanced Materials Research, Vols. 295-297, 2011, p. 2216-2222.

[20] Ren Y., Ruan J., Jia W. A. Output waveform analysis of an electro-hydraulic vibrator controlled by the multiple valves. Chinese Journal of Mechanical Engineering, Vol. 1, Issue 27, 2014, p. 186-197.

[21] Chiang M. H., Lin H. T. The leveling position control of a four-axial active pneumatic isolation system using PWM-driving parallel dual-on/off valves. Applied Mechanics and Materials, Vols. 110-116, 2012, p. 3176-3183.

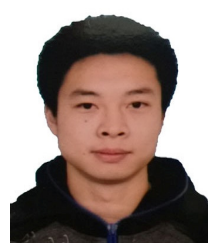

Zhen Meng received Master degree in School of Mechanical Engineering from Hangzhou Dianzi University, Hangzhou, China, in 2010. Now he is studying for a Doctor's degree from Zhejiang Sci-Tech University. His current research interests include hydraulic machine, dynamics and vibrational cutting.

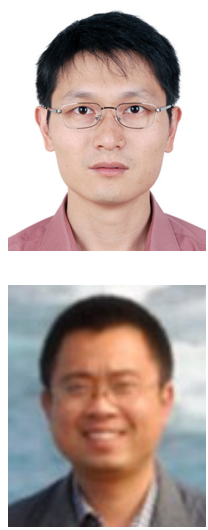

Chuanyu Wu received Ph.D. degree in College of Mechanical Engineering from Zhejiang University, Hangzhou, China, in 2003. Now he is vice-present of Mechanical Engineering and Automation Institute in Zhejiang Sci-Tech University. His current research interests include mechanism, robot technology and intelligent agricultural equipment.

Jing Ni received Ph.D. degree in College of Mechanical Engineering from Zhejiang University, Hangzhou, China, in 2006. Now he is vice-present of Mechanical Engineering Institute in Hangzhou Dianzi University. His current research interests include electro-hydraulic servo control, automation equipment and robot application. 